\title{
Upfront brain radiotherapy improves intracranial progression-free survival but not overall survival in lung adenocarcinoma patients with brain metastases: a retrospective, single-institutional analysis from China
}

\section{Aijun Jiang}

Shandong University

Meng Ni

Shandong Tumor Hospital and Institute

Li Li

Shandong Tumor Hospital and Institute

Fen Zhao

Shandong Tumor Hospital and Institute

Yuanhu Yao

Xuzhou Medical College Affiliated Hospital

Xin Ding

Xuzhou Medical College Affiliated Hospital

Qingxi Yu

Shandong Tumor Hospital and Institute

Longzhen Zhang

Xuzhou Medical College Affiliated Hospital

Shuanghu Yuan ( $\square$ yuanshuanghu@sina.com )

Shandong University https://orcid.org/0000-0002-8327-2524

\section{Research}

Keywords: brain metastases, lung adenocarcinoma, epidermal growth factor receptor tyrosine kinase inhibitor, brain radiotherapy

Posted Date: October 23rd, 2020

DOl: https://doi.org/10.21203/rs.3.rs-40353/v2 
License: (c) (i) This work is licensed under a Creative Commons Attribution 4.0 International License. Read Full License

Version of Record: A version of this preprint was published at Journal of Cancer on January 1st, 2022. See the published version at https://doi.org/10.7150/jca.64335. 


\section{Abstract}

Aims: The optimal timing of brain radiotherapy (BRT) for lung adenocarcinoma patients with brain metastases (BM) remains controversial. In this retrospective study, we performed a retrospective review to investigate the differential benefit of upfront versus deferred BRT for lung adenocarcinoma patients with BM.

Methods: A total of 354 lung adenocarcinoma patients with BM treated in the Affiliated Cancer Hospital of Shandong University met the inclusion criteria for the study. Patients were divided into two groups: upfront BRT and deferred BRT. Intracranial progression-free survival (iPFS) and overall survival (OS) were measured from the date of brain metastases. Subgroup analyses according to gene mutation status were also performed.

Results: Among the entire cohort, the median iPFS with upfront BRT (16.3 months) was longer than that with deferred BRT (11.3 months, $p=0.001)$. However, the median OS did not differ significantly between patients who received upfront BRT and deferred BRT (27.6 and 31.5 months, respectively, $p=0.813$ ). Subgroup analyses indicated that upfront BRT yielded a significantly longer iPFS than deferred BRT $(p=0.003)$ only for patients without EGFR gene mutation. In all subgroups, the median OS showed no significant difference between upfront BRT and deferred BRT.

Conclusion: This single-institutional retrospective study showed that in patients with lung adenocarcinoma and BM, upfront BRT was associated with a significantly longer iPFS but no improvement in OS compared with deferred BRT. Considering the neurocognitive toxicities of BRT previously reported in the literature, deferred BRT might be considered as an acceptable therapeutic option for the treatment of patients with lung adenocarcinoma and BM.

\section{Introduction}

Non-small cell lung cancer (NSCLC) accounts for about $87 \%$ of all lung cancer cases ${ }^{\left[{ }^{[1]}\right]}$ and $20-40 \%$ of NSCLC patients will develop brain metastases $(B M)^{[2],[3]}$. The proportion of adenocarcinoma among NSCLC cases has continued to increase in recent years ${ }^{[1]}$. In Asians, $~ 50 \%$ of lung adenocarcinomas harbor epidermal growth factor receptor (EGFR) mutation $s^{[4]}$. EGFR mutation status is highly concordant between primary NSCLC and corresponding $\mathrm{BM}^{[5]}$. Chemotherapy for such BM is not an effective treatment because of the existence of the blood-brain-barrier (BBB) ${ }^{[6]}$. For decades whole brain radiotherapy (WBRT) has been considered the standard care for patients with $\mathrm{BM}$, which is associated with an overall response rate of $60 \%$, a 6 -month disease control rate of $50 \%$ and a median overall survival (OS) of 4-6 months $s^{[7],[8],[9]}$.

During the past decade, the advancement of EGFR tyrosine kinase inhibitors (EGFR-TKIs) has revolutionarily transformed the landscape of treatment and prognosis of advanced lung adenocarcinoma patients ${ }^{[10]]}$. EGFR-TKIs were demonstrated to be safe and significantly efficacious in EGFR mutated 
NSCLC patients with BM, leading to a median progression-free survival of 14.5 months, a median OS of 21.9 month $s^{[111]}$ and intracranial disease response rates of $75-88 \%[[1],[13],[14]$. Among patients with previously untreated advanced NSCLC with EGFR mutation, osimertinib yielded a longer OS of 38.6 months than a comparator EGFR-TKI (31.8 months)[[15]]. EGFR TKIs are recommended as first line systemic therapy for patients with metastatic NSCLC harboring EGFR activating mutations ${ }^{[16],[17]}$.

Interestingly, a recent study demonstrated that patients with EGFR-mutated NSCLC are more sensitive to

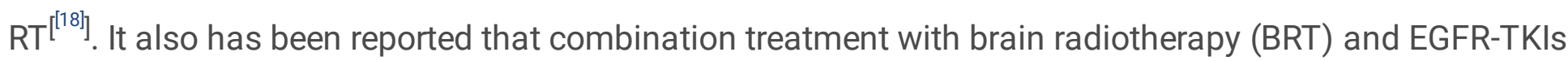
presents superior response rate and disease control rate compared with BRT or TKI alone ${ }^{[19],[20]]}$. However, the retrospective study by Magnuson et al ${ }^{[21]}$ demonstrated inferior OS with deferral of BRT. Another study reported that upfront BRT followed by TKI therapy may be an appropriate initial management approach for EGFR-mutant NSCLC patients with BM ${ }^{[22]}$.

However, in contrast to the conclusions of Magnuson et al, a retrospective analysis demonstrated that the addition of WBRT to EGFR-TKIs, compared with TKIs alone, did not improve the intracranial progressionfree survival (iPFS) and led to a worse OS in NSCLC with EGFR mutation and BM ${ }^{[23]}$. A study in China also suggested that upfront WBRT can be safely delayed in EGFR-mutant lung cancer with $\mathrm{BM}^{\left[{ }^{[24]}\right]}$. In the European Society of Medical Oncology guide-lines for metastatic NSCLC, delay of upfront WBRT is considered in NSCLC patients with minimal and asymptomatic BM.

Thus, there is no consensus on the management of patients with lung adenocarcinoma and BM, and specifically, the optimal timing of BRT has yet to be determined. In the present retrospective study, we investigated the differential benefit of upfront versus deferred BRT for lung adenocarcinoma patients with BM. We also performed subgroup analyses according to gene mutation status.

\section{Materials And Methods}

\section{Study design and patient selection}

This study was approved by our institutional review board. Through a review of hospital records, we identified 776 lung adenocarcinoma patients with BM treated at the Affiliated Cancer Hospital of Shandong University between June 1, 2013 and October 31, 2016. The eligibility criteria for this study were: (1) lung adenocarcinoma with BM, and (2) treatment with linear accelerator-based BRT. The exclusion criteria were: (1) upfront use of a TKI before BM diagnosis; (2) failure to complete the BRT plan; (3) meningeal metastases; (4) surgical resection or stereotactic radiosurgery (SRS); (5) presence of other primary tumors; (6) history of BRT; and (7) < 3 months of follow-up. The included patients were divided into two groups: upfront BRT and deferred BRT. Upfront BRT was defined as BRT within 2 months after diagnosis of BM. Tumor response was assessed every 2-3 months according to response evaluation criteria in solid tumors (RECIST) 1.1[[25]] by contrast-enhanced magnetic resonance imaging (MRI) or computed tomography (CT). 
The last date of follow-up was March 31, 2018. The following variables were collected at BM diagnosis: (1) age; (2) gender; (3) smoking status; (4) Karnofsky performance status (KPS) ; (5) histology; (6) gene mutation status; (7) extracranial metastases; (8) size of largest BM; (9) number of BMs; (10) symptoms of BMs; (11) radiotherapy mode; and (12) treatments. A disease-specific Graded Prognostic Assessment (DS-GPA) was calculated. The radiotherapy modes were divided into three groups: WBRT, LBRT (local brain radiotherapy) and WBRT + LBRT. The gross tumor area radiotherapy was named as LBRT. The equivalent dose in $2 \mathrm{~Gy} /$ fraction (EQD2) of WBRT was calculated to be $32.5-50$ Gy based on the LQ model. The accumulated LBRT dose was 50-60Gy with 2-4Gy per fraction and one fraction per day. Gene mutation positive was defined as EGFR+ (19 or 21) or ALK+ gene mutation. The dates of initial cancer diagnosis, BM diagnosis, BRT, systemic treatments, intracranial progression, most recent followup, and death were recorded. iPFS was defined as the time from BM diagnosis to intracranial progression, death, or last follow-up. OS was defined as the time from BM diagnosis to death or last follow-up.

\section{Statistical analysis}

Statistical comparisons between two groups were performed in SPSS, version 21.0 (SPSS Inc., Chicago, IL, USA). Comparisons of clinical characteristics between the upfront and deferred BRT groups were performed using the chi-square test. Kaplan-Meier analysis was used to estimate iPFS and OS and generate surviva1 curves, and log-rank testing was used to assess differences between the groups. Multivariable Cox proportional hazards analysis were performed according to backward stepwise regression. Differences were considered to be statistically significant if $p<0.05$.

\section{Results}

\section{Patient characteristics}

Table 1. Characteristics of 354 lung adenocarcinoma patients with BM 


\begin{tabular}{|c|c|c|c|c|c|c|}
\hline \multirow[b]{2}{*}{ Characteristic } & \multicolumn{2}{|c|}{ Upfront BRT( $n=273)$} & \multicolumn{3}{|c|}{ Deferred BRT $(n=81)$} & \multirow[b]{2}{*}{ p } \\
\hline & $\mathrm{n}$ & $\%$ & $\mathrm{n}$ & $\%$ & $c^{2}$ & \\
\hline Age & & & & & 0.133 & 0.716 \\
\hline$\leq 60$ years & 176 & 64.5 & 54 & 66.7 & & \\
\hline$\triangle 60$ years & 97 & 35.5 & 27 & 33.3 & & \\
\hline Gender & & & & & 2.327 & 0.127 \\
\hline Male & 151 & 55.3 & 37 & 45.7 & & \\
\hline Female & 122 & 44.7 & 44 & 54.3 & & \\
\hline Smoking status & & & & & 0.295 & 0.587 \\
\hline Current/former & 89 & 32.8 & 24 & 29.6 & & \\
\hline Never & 182 & 67.2 & 57 & 70.4 & & \\
\hline Symptomatic BM & & & 30 & & 18.773 & 0.000 \\
\hline Yes & 175 & 64.1 & 51 & 37.0 & & \\
\hline No & 98 & 35.9 & & 63.0 & & \\
\hline No. of BMs & & & & & 0.756 & 0.385 \\
\hline $1-3$ & 164 & 60.1 & 53 & 65.4 & & \\
\hline$\varangle 3$ & 109 & 39.9 & 28 & 34.6 & & \\
\hline Size of largest BM & & & & & 22.656 & 0.000 \\
\hline$\leq 1 \mathrm{~cm}$ & 74 & 27.1 & 45 & 55.6 & & \\
\hline$\nabla 1 \mathrm{~cm}$ & 199 & 72.9 & 36 & 44.4 & & \\
\hline Gene mutation & & & & & 2.673 & 0.263 \\
\hline Positive & 71 & 26.0 & 25 & 30.9 & & \\
\hline Negative & 56 & 20.5 & 21 & 25.9 & & \\
\hline Unknown & 146 & 53.5 & 35 & 43.2 & & \\
\hline Extracranial metastases & & & 59 & & & \\
\hline Yes & 153 & 56.0 & 22 & 72.8 & 7.335 & 0.007 \\
\hline No & 120 & 44.0 & & 27.2 & & \\
\hline DS-GPA & & & & & 0.123 & 0.726 \\
\hline $0-2$ & 166 & 60.8 & 51 & 63.0 & & \\
\hline
\end{tabular}




\begin{tabular}{|lcccccc|}
$2.5-4$ & 107 & 39.2 & 30 & 37.0 & & \\
BRT mode & & & & & 10.210 & 0.006 \\
WBRT & 120 & 44.0 & 48 & 59.3 & & \\
LBRT & 39 & 14.2 & 15 & 18.5 & & \\
WBRT + LBRT & 114 & 41.8 & 18 & 22.2 & & \\
\hline
\end{tabular}

Abbreviations: BM, brain metastases; DS-GPA, Diagnosis-Specific Graded Prognostic Assessment; BRT, brain radiotherapy; WBRT, whole brain radiotherapy; LBRT, local brain radiotherapy.

After application of the inclusion and exclusion criteria, a total of 354 patients were identified for survival analyses (273 who received upfront BRT and 81 who received deferred BRT). The patients' demographic and clinical characteristics are summarized in Table 1. The median follow-up for all patients was 23.0 months (range, 0.6-113.6 months). The median age at BM diagnosis for all patients was 57 years (range, 27-78 years). The median ages at BM diagnosis for the upfront BRT and deferred BRT groups were 57 and 55 years, respectively. Additionally, 188 (53\%) patients were male, and 89 patients (25\%) were smokers. In total, 96 patients $(27.1 \%$ ) displayed a positive gene mutation (EGFR+ or ALK+), 77 patients $(21.8 \%)$ displayed no positive gene mutation, and 181 patients $(51.1 \%)$ did not undergo genetic testing. Among the patients with a positive gene mutation, $92.7 \%$ patients $(89 / 96)$ received targeted therapy. However, $42.0 \%$ patients $(76 / 181)$ with unknown gene mutation status and $35.1 \%$ patients (27/77) without EGFR gene mutation also received targeted therapy.

Among all 354 patients, 168 patients (47.5\%) received WBRT, 54 (15.3\%) received local BRT (LBRT), and $132(37.3 \%)$ received both WBRT and LBRT. The median maximum diameter of the BMs was $15.5 \mathrm{~mm}$. Overall, $52.5 \%$ of patients $(186 / 354)$ had BM at primary diagnosis. Patients who received upfront BRT were more likely to have symptomatic BM $(64.1 \%$ vs. $37 \% ; p<0.001)$ and were more likely to have $B M s>1$ $\mathrm{cm}(72.9 \%$ vs. $44.4 \%$; $p<0.001)$. Patients who received upfront BRT were less likely to have extracranial metastases ( $56 \%$ vs. $72.8 \% ; p=0.007)$ and more likely to receive LBRT and WBRT ( $41.8 \%$ vs. $22.2 \% ; p=0.006$ ). No differences were observed between the two groups with respect to age, sex, Eastern Cooperative Oncology Group (ECOG) performance status, smoking status, number of BMs, gene mutation, or DS-GPA at the time of BM diagnosis.

\section{Survival outcomes for the entire cohort}

For the entire cohort, the median iPFS and OS were 14.6 months ( $95 \%$ confidence interval [Cl]: 12.4-16.7 months) and 28.2 months (95\% Cl: 24.7-31.7 months), respectively (Figure 1A and B). The median iPFS durations with upfront BRT and deferred BRT were 16.3 months (95\% Cl: 14.2-18.5 months) and 11.3 months (95\% Cl: 9.1-13.5 months), respectively. The median iPFS with upfront BRT was significantly longer than that with deferred BRT (log-rank $p=0.001$; Table 2). The impact of upfront BRT on iPFS 
remained significant on multivariable analysis $(\mathrm{p}=0.003$, hazard ratio $[\mathrm{HR}]=0.662[0.503-0.871])$. Interestingly, the median OS with upfront BRT (27.6 months, 95\% Cl: 23.9-31.3 months) appeared to be shorter than that with deferred BRT (31.5 months, 95\% Cl: 22.6-40.3 months), but the difference was not significant (log rank $\mathrm{p}=0.813$; Table 3 ). Propensity Score-Matched Analysis Propensity score matching was used to address potential treatment selection bias. These results were still unchanged after propensity score matching.

Table 2. Univariable and multivariable analyses of covariables associated with iPFS in NSCLC patients with BM

\begin{tabular}{|c|c|c|c|c|c|}
\hline \multirow[b]{2}{*}{ Variable } & \multicolumn{2}{|c|}{ Univariable } & \multicolumn{3}{|c|}{ Mutivariable } \\
\hline & $c^{2}$ & $\mathbf{p}$ & HR & $95 \% \mathrm{Cl}$ & p \\
\hline upfront BRT vs deferred BRT & 11.376 & 0.001 & 0.662 & $0.503-0.871$ & 0.003 \\
\hline 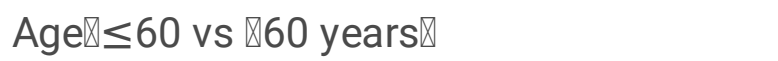 & 0.865 & 0.352 & & & \\
\hline Gender (male vs female) & 2.244 & 0.134 & & & \\
\hline Smoking status (never vs current/former) & 1.313 & 0.252 & & & \\
\hline Symptom (yes vs no) & 1.475 & 0.225 & & & \\
\hline No. of $\mathrm{BM}(1-3$ vs $\otimes 3)$ & 1.435 & 0.231 & & & \\
\hline Size of largest $B M(\leq 1 \mathrm{vs} \otimes 1 \mathrm{~cm})$ & 0.229 & 0.632 & & & \\
\hline \multicolumn{6}{|l|}{ Gene mutation } \\
\hline Positive vs negative & 4.051 & 0.044 & & & \\
\hline Positive vs unknown & 0.003 & 0.958 & & & \\
\hline Negative vs unknown & 4.018 & 0.045 & & & \\
\hline Extracranial metastases (yes vs no) & 12.466 & 0.000 & & & \\
\hline DS-GPA (2.5-4 vs $0-2)$ & 13.380 & 0.000 & 0.659 & $0.510-0.851$ & 0.001 \\
\hline \multicolumn{6}{|l|}{ BRT mode } \\
\hline LBRT vs WBRT & 0.224 & 0.636 & & & \\
\hline LBRT + WBRT vs WBRT & 3.885 & 0.049 & & & \\
\hline LBRT + WBRT vs LBRT & 1.070 & 0.301 & & & \\
\hline
\end{tabular}

Abbreviations: BM, brain metastases; DS-GPA, Diagnosis-Specific Graded Prognostic Assessment; BRT, brain radiotherapy; WBRT, whole brain radiotherapy; LBRT, local brain radiotherapy. 
Table 3. Univariable and multivariable analyses of covariables associated with OS in NSCLC patients with BM

\begin{tabular}{|c|c|c|c|c|c|}
\hline \multirow[b]{2}{*}{ Variable } & \multicolumn{2}{|c|}{ Univariable } & \multicolumn{3}{|c|}{ Mutivariable } \\
\hline & $c^{2}$ & $\mathbf{p}$ & HR & $95 \% \mathrm{Cl}$ & $\mathbf{p}$ \\
\hline Upfront BRT vs deferred BRT & 0.056 & 0.813 & & & \\
\hline 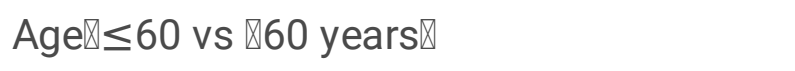 & 0.027 & 0.869 & & & \\
\hline Gender (male vs female) & 4.259 & 0.039 & & & \\
\hline Smoking status (never vs current/former) & 4.517 & 0.034 & 0.651 & $0.489-0.865$ & 0.003 \\
\hline Symptom (yes vs no) & 0.532 & 0.466 & & & \\
\hline No. of $B M(1-3$ vs $\otimes 3)$ & 7.141 & 0.008 & 0.678 & $0.515-0.892$ & 0.006 \\
\hline Size of largest $B M(\leq 1$ vs $\nabla 1 \mathrm{~cm})$ & 3.318 & 0.069 & 0.730 & $0.546-0.976$ & 0.034 \\
\hline \multicolumn{6}{|l|}{ Gene mutation } \\
\hline Positive vs negative & 3.869 & 0.049 & 0.660 & $0.442-0.985$ & 0.042 \\
\hline Positive vs unknown & 4.300 & 0.038 & 0.637 & $0.455-0.893$ & 0.009 \\
\hline Negative vs unknown & 0.012 & 0.914 & & & \\
\hline Extracranial metastases (yes vs no) & 14.194 & 0.000 & 1.749 & $1.314-2.330$ & 0.000 \\
\hline DS-GPA (2.5-4 vs 0-2) & 9.740 & 0.002 & & & \\
\hline \multicolumn{6}{|l|}{ BRT mode } \\
\hline LBRT vs WBRT & 0.679 & 0.410 & & & \\
\hline LBRT + WBRT vs WBRT & 3.198 & 0.074 & & & \\
\hline LBRT + WBRT vs LBRT & 0.185 & 0.667 & & & \\
\hline
\end{tabular}

Abbreviations: BM, brain metastases; DS-GPA, Diagnosis-Specific Graded Prognostic Assessment; BRT, brain radiotherapy; WBRT, whole brain radiotherapy; LBRT, local brain radiotherapy.

\section{Subgroup analyses of patients with EGFR mutation}

According to the gene mutation status, we performed further analyses by dividing the patients into three groups: EGFR mutation-positive group (positive), no EGFR gene mutation group (negative), and no genetic testing group (unknown). Patients with ALK, ROS1, or c-MET mutation (5 patients) were excluded from the EGFR mutation-positive group. For patients with EGFR mutation, the median iPFS and OS were 17.1 months (95\% Cl: 13.7-20.5 months) and 34.4 months (95\% Cl: 28.0-40.9 months), respectively. With upfront BRT and deferred BRT, the median iPFS and OS were 18.0 and 14.2 months $\left(c^{2}=2.222\right.$, 
$p=0.136)$ and 34.4 and 34.8 months $\left(c^{2}=0.006, p=0.938\right)$, respectively. Univariate analysis indicated that upfront BRT and WBRT+LBRT were associated with iPFS, but the differences were not significant. Upfront BRT was not significantly associated with OS according to the univariate and multivariate analyses (Appendix Table 4 and Table 5, not shown).

\section{Subgroup analyses for patients without genetic testing results}

For patients without genetic testing, the median iPFS and OS were 16.3 months ( $95 \% \mathrm{Cl}$ : 13.3-19.3 months) and 26.1 months (95\% Cl: 22.1-30.1 months), respectively. With upfront BRT and deferred BRT, the median iPFS and OS for these patients were 14.9 and 17.4 months $\left(c^{2}=1.909, p=0.167\right)$ and 24.8 and 35.7 months $\left(c^{2}=0.652, p=0.420\right)$, respectively. The univariate and multivariate analyses identified gender $(p=0.027, H R=1.419[1.047-2.125])$, extracranial metastases $(p=0.042, H R=1.627[1.018-2.599])$, and DSGPA ( $p=0.005, H R=0.512[0.309-0.816])$ as significantly associated with iPFS. Upfront BRT was not significantly associated with iPFS or OS in these patients according to the univariate and multivariate analyses. Univariate analysis identified gender $\left(c^{2}=4.914, p=0.027\right)$, smoking status $\left(c^{2}=4.678, p=0.031\right)$, extracranial metastases $\left(c^{2}=14.325, p=0.000\right)$, and DS-GPA $\left(c^{2}=11.288, p=0.001\right)$ as significantly associated with OS. On multivariate analysis, smoking status $(p=0.012, H R=0.623[0.432-0.899])$ and extracranial metastases $(\mathrm{p}=0.000, \mathrm{HR}=2.142[1.457-3.150])$ remained significantly associated with $\mathrm{OS}$ (Appendix Table 6 and Table 7, not shown).

\section{Subgroup analyses for patients without EGFR gene mutation}

For patients without EGFR gene mutation, the median iPFS and OS were 10.8 (95\% Cl: 9.3-12.4) and 23.4 months (95\% Cl: 19.0-27.9), respectively. Upfront BRT in these patients provided a longer median iPFS than deferred BRT (12.3 vs 8.5 months, $c^{2}=9.682, p=0.002$ ). However, no difference in the median OS (23.4 vs. 23.4 months, $c^{2}=0.137, p=0.711$ ) was observed between the patient who received upfront BRT and deferred BRT. On multivariate analysis, only upfront BRT yielded a longer iPFS than deferred BRT $(p=0.003, H R=0.412[0.231-0.733])$. However, no correlation between upfront BRT and OS was found on univariate and multivariate analyses. Multivariate analysis identified only the number of BMs as significantly associated with OS ( $p=0.051, \mathrm{HR}=0.570[0.324-1.002]$ ) (Appendix Table 8 and Table 9, not shown).

\section{Discussion}

Among NSCLC subtypes, adenocarcinoma is more prone to BM, and the prognosis and treatment strategy for lung adenocarcinoma patients are different from those of patients with other lung cancer types, especially for patients with EGFR gene mutations. The upfront BRT strategy has advantages and disadvantages. Although previous studies indicated that upfront BRT may be associated with better local control and longer OS, use of TKIs alone initially avoids the side effects of BRT. Two small randomized studies reported that the timing of WBRT did not influence OS in unselected NSCLC patients with BM receiving chemotherapy ${ }^{[26],[27]}$. Upfront SRS did not improve OS in oligo-brain metastases NSCLC patients 
compared with upfront chemotherapy ${ }^{\left[{ }^{[28]}\right]}$. The QUARTZ study ${ }^{[29]}$ indicated that WBRT provided little additional clinically significant benefit for BM patients with a KPS of $<70$. Use of an EGFR-TKI alone for BM in EGFR-mutant lung cancer patients showed outcomes comparable to those who received upfront RT followed by EGFR-TKI therapy ${ }^{[30]}$. The BRAIN study also suggested that upfront BRT can be safely delayed in EGFR-mutant lung cancer patients with $\mathrm{BM}^{[24]}$. Thus, to date, the value and timing of $\mathrm{BRT}$ for patients with BM from lung adenocarcinoma have remained controversial, especially for EGFR-mutant patients.

To the best of our knowledge, this study was the first retrospective analysis from China to compare survival outcomes with upfront and deferred BRT in patients with BM from lung adenocarcinoma. The targeted population in this study was lung adenocarcinoma patients with BM regardless of number of BMs, which was different from the population of the BRAIN study ${ }^{[24]}$. Among the entire cohort, the median iPFS was longer in patients who received upfront BRT than in those who received deferred BRT. However, in our subgroup analysis according to gene mutation status, iPFS no longer significantly differed with upfront versus deferred BRT for patients with EGFR gene mutation. Additionally, no significant differences in OS were observed between upfront and deferred BRT in all subgroups. Therefore, upfront BRT may offer an iPFS benefit in patients with BM from lung adenocarcinoma, especially in patients without EGFR gene mutation, but cannot provide an additional OS benefit.

Several qualitative reviews have suggested that TKI therapy may be used first before BRT in EGFR+ NSCLC patients with asymptomatic BM ${ }^{[3],[32],[33],[34]}$. However, Magnuson et al. ${ }^{[21]}$ reported that the use of upfront EGFR-TKI and deferral of RT was associated with inferior OS in patients with EGFR-mutant NSCLC who developed BM. For EGFR-mutant BM patients, our subgroup results showed that upfront BRT may provide a longer iPFS compared to deferred BRT, but the difference was not significant. On the other hand, upfront BRT was not significantly associated with OS according to the univariate and multivariate analysis.

In the present study, upfront BRT was defined as BRT within 2 months after diagnosis of BM, which was not consistent with the definition used in several previous studies ${ }^{[21,23,30]}$. Although $S R S$ is recommended by guidelines as a standard radiotherapy method for patients with 1-3 BMs, it is not available for most RT units in China. Therefore, patients treated with SRS were excluded from the present study. Due to the lack of randomization, the presence of bias was inevitable. Furthermore, the data from the subgroup analysis comparing the two treatment groups were insufficient due to the small number of patients treated with deferred BRT. The quality of evidence supporting these findings is low. In addition, the adverse events, quality of life and cognitive function could not be assessed due to the lack of clinical data. Moreover, the patients involved in the present study were all Chinese; hence, further investigations in patients of other races are required.

\section{Conclusions}


In summary, this single-institutional, retrospective study showed that in patients with lung adenocarcinoma and BM, upfront BRT was associated with a significantly longer iPFS, especially in patients without EGFR gene mutation. No difference in OS was observed between patients treated with upfront BRT versus deferred BRT. Considering the neurocognitive toxicities previously reported with upfront BRT, deferred BRT might be considered as an acceptable therapeutic option for the treatment of patients with lung adenocarcinoma and BMs.

\section{Abbreviations}

NSCLC, non-small cell lung cancer; BM, brain metastases; epidermal growth factor receptor tyrosine kinase inhibitors (EGFR-TKIs); BBB, blood-brain-barrier; RT, brain radiotherapy; BRT, brain radiotherapy; iPFS, intracranial progression-free survival; OS, overall survival; WBRT, whole brain radiotherapy; KPS, Karnofsky performance status; DS-GPA, Diagnosis-Specific Graded Prognostic Assessment; WBRT, whole brain radiotherapy; LBRT, local brain radiotherapy. SRS, stereotactic radiosurgery.

\section{Declarations}

\section{Funding}

This study was partially supported by the Shandong Key Research and Development Plan (grant numbers 2017CXGC1209 and 2017GSF18164), the Outstanding Youth Natural Science Foundation of Shandong Province (grant number JQ201423), the Jinan Clinical Medicine Science and Technology Innovation Plan (grant number 201704095), NSFC $₫$ grant number $81372413 \rrbracket$, NSFC $₫$ grant number $81671785 \rrbracket$, the Natural Science Foundation of Shandong Province (grant number 2016ZRC03118), the National Key Research and Development Program of China (grant number 2016YFC0904700), and the Project of Invigorating Health Care through Science, Technology and Education (grant number CXTDA2017034).

\section{Availability of data and materials}

Not applicable.

\section{Authors'contributions}

Conception and design: Shuanghu Yuan

Provision of study materials or patients: Shuanghu Yuan

Collection and assembly of data: Aijun Jiang

Data analysis and interpretation: Aijun Jiang

Manuscript writing: Aijun Jiang

Final approval of manuscript: All authors 
Accountable for all aspects of the work: All authors

\section{Ethics approval and consent to participate}

The clinical research committee of the study institute approved the study protocol, and written informed consent was waived by the Institutional Review Board.

\section{Consent for publication}

Not applicable.

\section{Competing interests}

The authors declare that they have no competing interests.

\section{Acknowledgements}

Not applicable.

\section{References}

[[1]] Lee DS, Kim YS, Kay CS, Kim SH, Yeo CD, Kim JW, et al. Distinctive Patterns of Initially Presenting Metastases and Clinical Outcomes According to the Histological Subtypes in Stage IV Non-Small Cell Lung Cancer. Medicine (Baltimore). 2016;95(6):e2795.

[[2]] Zhao J, Chen M, Zhong W, Zhang L, Li L, Xiao Y, et al. Cerebrospinal fluid concentrations of gefitinib in patients with lung adenocarcinoma. Clin Lung Cancer. 2013;14:188-93.

[[3]] Brower JV, Robins HI. Erlotinib for the treatment of brain metastases in non-small cell lung cancer. Expert Opin Pharmacother. 2016;17(7):1013-21.

[[4]] Suda K, Mitsudomi T, Shintani Y, Okami J, Ito H, Ohtsuka T, et al. Clinical impacts of EGFR mutation status: analysis of 5,780 surgically resected lung cancer cases. Ann Thorac Surg. 2020;29(6):S00034975(20)30982-6.

[[5]] Luo D, Ye X, Hu Z, Peng K, Song Y, Yin X, et al. EGFR mutation status and its impact on survival of Chinese non-small cell lung cancer patients with brain metastases. Tumour Biol. 2014;35(3):2437-44.

[[6]] Dagogo-Jack I, Gill CM, Cahill DP, Santagata S, Brastianos PK. Treatment of brain metastases in the modern genomic era. Pharmacol Ther. 2017;170:64-72.

[[7]] Arrieta O, Villarreal-Garza C, Zamora J, Blake-Cerda M, de la Mata MD, Zavala DG, et al. Long-term survival in patients with non-small cell lung cancer and synchronous brain metastasis treated with wholebrain radiotherapy and thoracic chemoradiation. Radiat Oncol. 2011;6:166. 
[[8]] Gaspar LE, Mehta MP, Patchell RA, Burri SH, Robinson PD, Morris RE, et al. The role of whole brain radiation therapy in the management of newly diagnosed brain metastases: a systematic review and evidence-based clinical practice guideline. Journa1 of neuro-oncology. 2010;96(1):1732.

[[9]] Khuntia D, Brown P, Li J, Mehta MP. Whole-brain radiotherapy in the management of brain metastasis. J Clin Oncol. 2006;24(8):1295-304.

[[10]] Tang N, Guo J, Zhang Q, Wang Y, Wang Z. Greater efficacy of chemotherapy plus bevacizumab compared to chemo- and targeted therapy alone on non-small cell lung cancer patients with brain metastasis. Oncotarget. 2015;7:3635-44.

[[11]] luchi T, Shingyoji M, Sakaida T, Hatano K, Nagano O, Itakura M, et al. Phase II trial of gefitinib alone without radiation therapy for Japanese patients with brain metastases from EGFR-mutant lung adenocarcinoma. Lung Cancer 2013;82:282-7.

[[12]] Park SJ, Kim HT, Lee DH, Kim KP, Kim SW, Suh C, et al. Efficacy of epidermal growth factor receptor tyrosine kinase inhibitors for brain metastasis in non-small cell lung cancer patients harboring either exon 19 or 21 mutation. Lung Cancer. 2012;77(3):556-60.

[[13]] Wu YL, Zhou C, Cheng Y, Lu S, Chen GY, Huang C, et al. Erlotinib as second-line treatment in patients with advanced non-small-cell lung cancer and asymptomatic brain metastases: a phase II study (CTONG0803). Ann Oncol. 2013;24(4):993-9.

[[14]] Mamon HJ, Yeap BY, Jänne PA, Reblando J, Shrager S, Jaklitsch MT, et al. High risk of brain metastases in surgically staged IIIA non-small-cell lung cancer patients treated with surgery, chemotherapy, and radiation. J Clin Oncol. 2005;23(7):1530-7.

[[15]] Ramalingam SS, Vansteenkiste J, Planchard D, Cho BC, Gray JE, Ohe Y, et al. Overall Survival with Osimertinib in Untreated, EGFR-Mutated Advanced NSCLC. N Engl J Med. 2020;382(1):41-50.

[[16]] Planchard D, Popat S, Kerr K, Novello S, Smit EF, Faivre-Finn C, et al. Correction to: "Metastatic nonsmall cell lung cancer: ESMO Clinical Practice Guidelines for diagnosis, treatment and follow-up". Ann Oncol. . 2019;30(5):863-70.

[[17]] Tsakonas G, De Petris L, Ekman S. Management of brain metastasized non-small cell lung cancer (NSCLC) - From local treatment to new systemic therapies. Cancer Treat Rev. 2017;54:122-31.

[[18]] Johung KL, Yao X, Li F, Yu JB, Gettinger SN, Goldberg S, et al. A clinical model for identifying radiosensitive tumor genotypes in non-small cell lung cancer. Clin Cancer Res 2013;19:5523-32.

[[19]] Jiang T, Min W, Li Y, Yue Z, Wu C, Zhou C. Radiotherapy plus EGFR TKIs in non-small cell lung cancer patients with brain metastases: an update meta-analysis. Cancer Med 2016;5:1055-65. 
[[20]] Chen CH, Lee HH, Chuang HY, Hung JY, Huang MY, Chong IW. Combination of Whole-Brain Radiotherapy with Epidermal Growth Factor Receptor Tyrosine Kinase Inhibitors Improves Overall Survival in EGFR-Mutated Non-Small Cell Lung Cancer Patients with Brain Metastases. Cancers (Basel). 2019;11(8):1092.

[[21]] Magnuson WJ, Lester-Coll NH, Wu AJ, Yang TJ, Lockney NA, Gerber NK, et al. Management of Brain Metastases in Tyrosine Kinase Inhibitor-Naïve Epidermal Growth Factor Receptor-Mutant Non-Small-Cell Lung Cancer: A Retrospective Multi-Institutional Analysis. J Clin Oncol. 2017;35(10):1070-7.

[[22]] Saruwatari K, Ikeda T, Saeki S, Shingu N, Imamura K, Komatu T, et al. Upfront Cranial Radiotherapy Followed by Erlotinib Positively Affects Clinical Outcomes of Epidermal Growth Factor Receptor-mutant Non-small Cell Lung Cancer With Brain Metastases. Anticancer Res. 2019;39(2):923-31.

[[23]] Jiang T, Su C, Li X, Zhao C, Zhou F, Ren S, et al. EGFR TKIs plus WBRT Demonstrated No Survival Benefit Other Than That of TKIs Alone in Patients with NSCLC and EGFR Mutation and Brain Metastases. J Thorac Oncol. 2016;11(10):1718-28.

[[24]] Yang JJ, Zhou C, Huang Y, Feng J, Lu S, Song Y, et al. Icotinib versus whole-brain irradiation in patients with EGFR-mutant non-small-cell lung cancer and multiple brain metastases (BRAIN): a multicentre, phase 3, open-label, parallel, randomised controlled trial. Lancet Respir Med. 2017;5(9):70716.

[[25]] Eisenhauer EA, Therasse P, Bogaerts J, Schwartz LH, Sargent D, Ford R, et al. New response evaluation criteria in solid tumours: Revised RECIST guideline (version 1.1). Eur J Cancer 2009; 45:22847.

[[26]] Robinet G, Thomas P, Breton JL, Léna H, Gouva S, Dabouis G, et al. Results of a phase III study of early versus delayed whole brain radiotherapy with concurrent cisplatin and vinorelbine combination in inoperable brain metastasis of non-small cell lung cancer: Groupe Francais de Pneumo-Cancerologie (GFPC) Protocol 95-1. Ann Oncol 2001;12:59-67.

[[27]] Lee DH, Han JY, Kim HT, Yoon SJ, Pyo HR, Cho KH, et al. Primary chemotherapy for newly diagnosed non-small cell lung cancer patients with synchronous brain metastases compared with whole-brain radiotherapy administered first. Cancer 2008;113:143-9.

[[28]] Lim SH, Lee JY, Lee MY, Kim HS, Lee J, Sun JM, et al. A randomized phase III trial of stereotactic radiosurgery (SRS) versus observation for patients with asymptomatic cerebral oligo-metastases in nonsmall-cell lung cancer. Ann Oncol. 2015;26(4):762-8.

[[29]] Mulvenna P, Nankivell M, Barton R, Faivre-Finn C, Wilson P, McColl E, et al. Dexamethasone and supportive care with or without whole brain radiotherapy in treating patients with non-small cell lung cancer with brain metastases unsuitable for resection or stereotactic radiotherapy (QUARTZ): results from a phase 3, non-inferiority, randomised trial. Lancet. 2016;388(10055):2004-14. 
[[30]] Hyun DG, Choi CM, Lee DH, Kim SW, Yoon S, Kim WS, et al. Outcomes according to initial and subsequent therapies following intracranial progression in patients with EGFR-mutant lung cancer and brain metastasis. PLoS One. 2020;15(4):e0231546.

[[31]] Jamal-Hanjani M, Spicer J. Epidermal growth factor receptor tyrosine kinase inhibitors in the treatment of epidermal growth factor receptor-mutant non-small cell lung cancer metastatic to the brain. Clin Cancer Res. 2012;18(4):938-44.

[[32]] Bartolotti M, Franceschi E, Brandes AA. EGF receptor tyrosine kinase inhibitors in the treatment of brain metastases from non-small-cell lung cancer. Expert Rev Anticancer Ther. 2012;12(11):1429-35.

[[33]] Zimmermann S, Dziadziuszko R, Peters S. Indications and limitations of chemotherapy and targeted agents in non-small cell lung cancer brain metastases. Cancer Treat Rev. 2014;40(6):716-22.

[[34]] Soon YY, Leong CN, Koh WY, Tham IW. EGFR tyrosine kinase inhibitors versus cranial radiation therapy for EGFR mutant non-small cell lung cancer with brain metastases: a systematic review and meta-analysis. Radiother Oncol. 2015;114(2):167-72.

Il cell lung cancer: Groupe Francais de Pneumo-Cancerologie (GFPC) Protocol 95-1. Ann Oncol 2001;12:59-67.

[[27]] Lee DH, Han JY, Kim HT, Yoon SJ, Pyo HR, Cho KH, et al. Primary chemotherapy for newly diagnosed non-small cell lung cancer patients with synchronous brain metastases compared with whole-brain radiotherapy administered first. Cancer 2008;113:143-9.

[[28]] Lim SH, Lee JY, Lee MY, Kim HS, Lee J, Sun JM, et al. A randomized phase III trial of stereotactic radiosurgery (SRS) versus observation for patients with asymptomatic cerebral oligo-metastases in nonsmall-cell lung cancer. Ann Oncol. 2015;26(4):762-8.

[[29]] Mulvenna P, Nankivell M, Barton R, Faivre-Finn C, Wilson P, McColl E, et al. Dexamethasone and supportive care with or without whole brain radiotherapy in treating patients with non-small cell lung cancer with brain metastases unsuitable for resection or stereotactic radiotherapy (QUARTZ): results from a phase 3, non-inferiority, randomised trial. Lancet. 2016;388(10055):2004-14.

[[30]] Hyun DG, Choi CM, Lee DH, Kim SW, Yoon S, Kim WS, et al. Outcomes according to initial and subsequent therapies following intracranial progression in patients with EGFR-mutant lung cancer and brain metastasis. PLoS One. 2020;15(4):e0231546.

[[31]] Jamal-Hanjani M, Spicer J. Epidermal growth factor receptor tyrosine kinase inhibitors in the treatment of epidermal growth factor receptor-mutant non-small cell lung cancer metastatic to the brain. Clin Cancer Res. 2012;18(4):938-44.

[[32]] Bartolotti M, Franceschi E, Brandes AA. EGF receptor tyrosine kinase inhibitors in the treatment of brain metastases from non-small-cell lung cancer. Expert Rev Anticancer Ther. 2012;12(11):1429-35. 
[[33]] Zimmermann S, Dziadziuszko R, Peters S. Indications and limitations of chemotherapy and targeted agents in non-small cell lung cancer brain metastases. Cancer Treat Rev. 2014;40(6):716-22.

[[34]] Soon YY, Leong CN, Koh WY, Tham IW. EGFR tyrosine kinase inhibitors versus cranial radiation therapy for EGFR mutant non-small cell lung cancer with brain metastases: a systematic review and meta-analysis. Radiother Oncol. 2015;114(2):167-72.

\section{Figures}
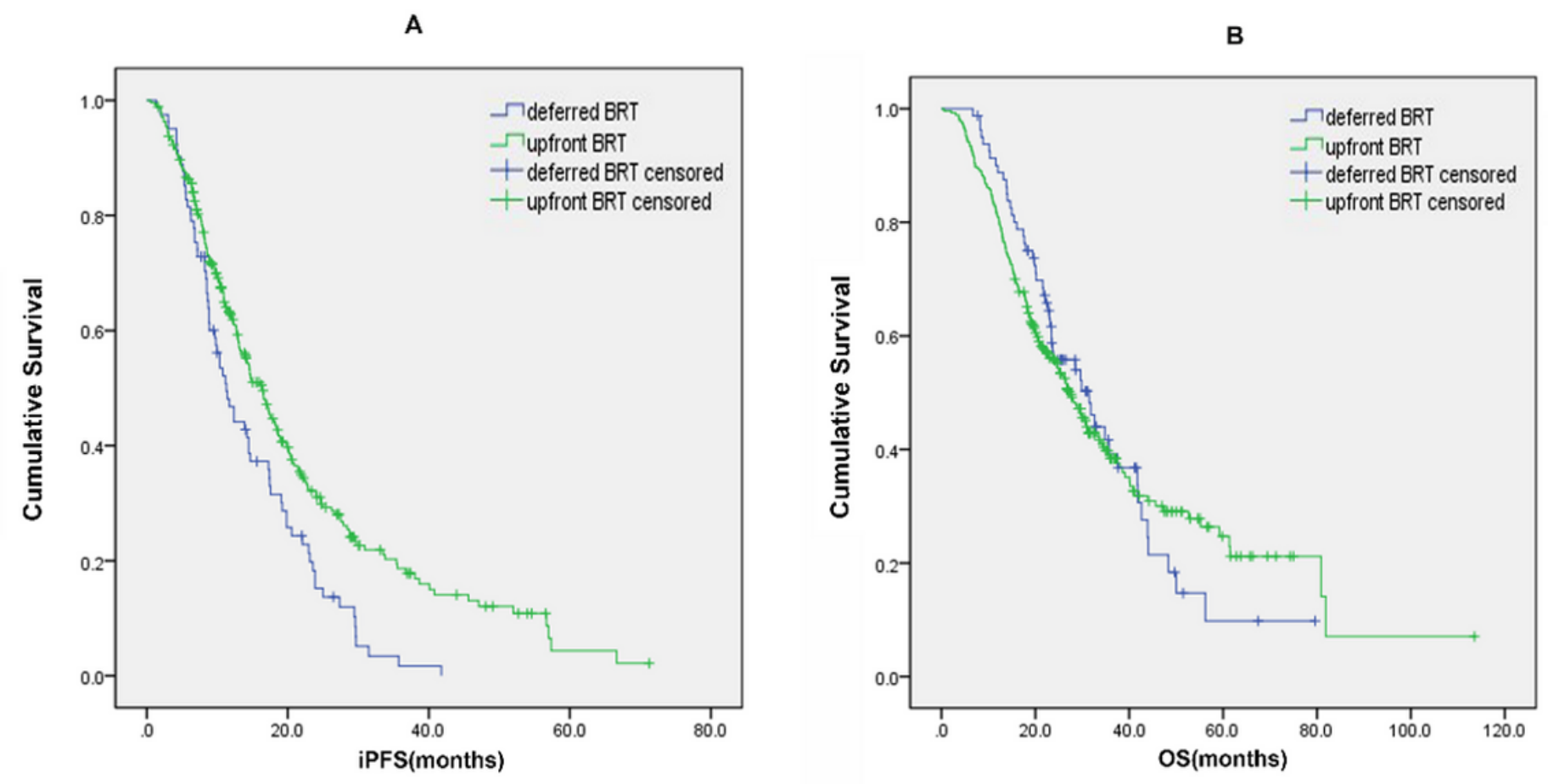

Figure 1

Kaplan-Meier survival curves of iPFS (A) and OS (B) for the entire cohort. Abbreviations: BRT, brain radiotherapy; iPFS, intracranial progression-free survival; OS, overall survival. 
A

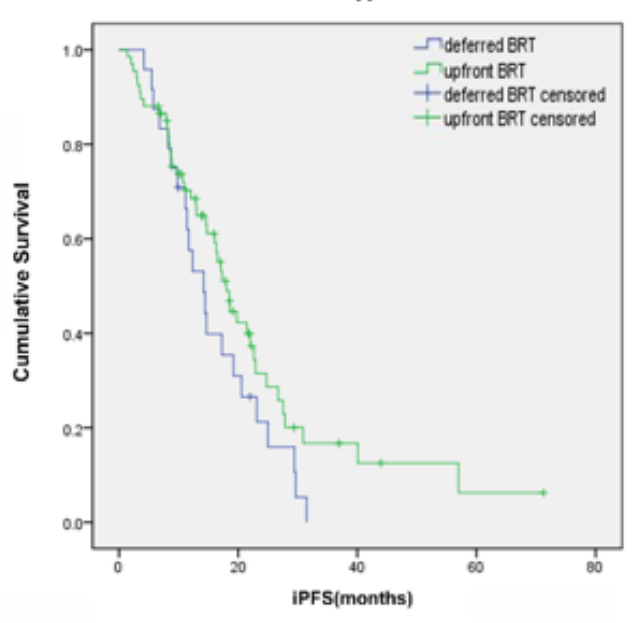

c

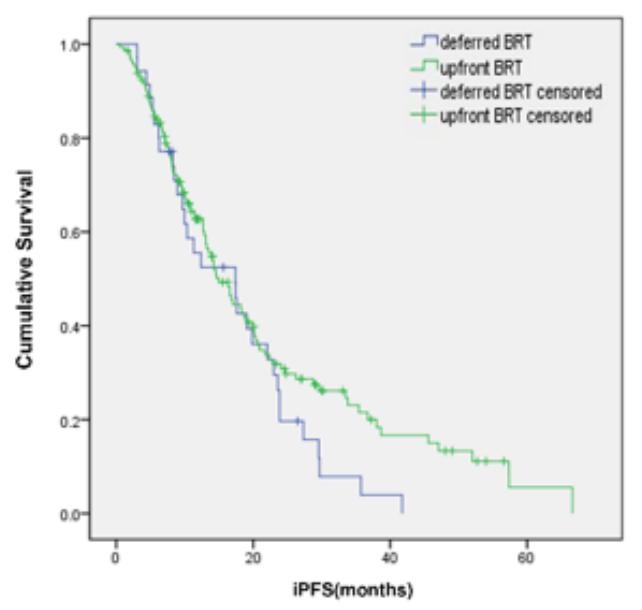

E

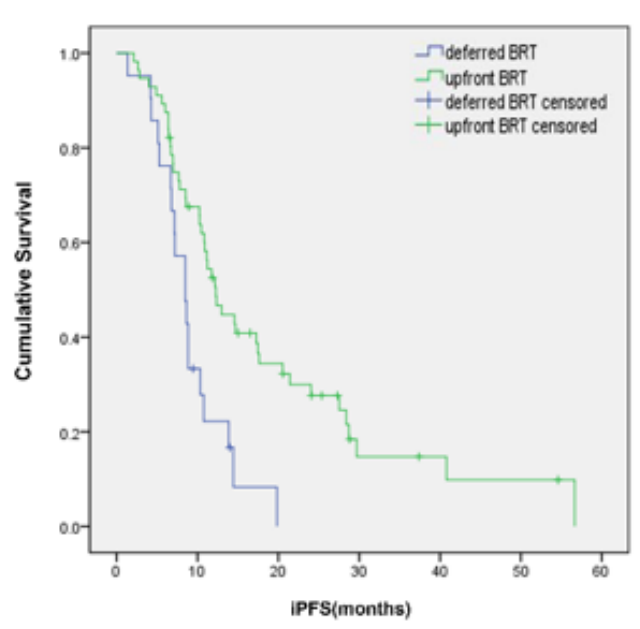

B

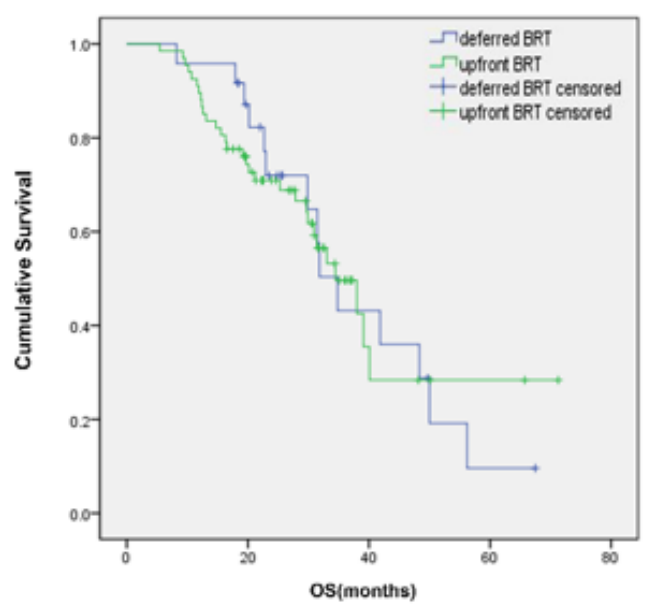

D

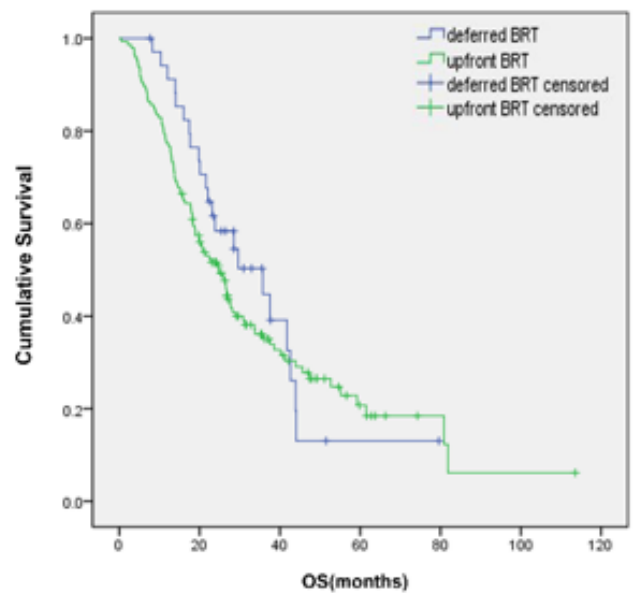

F

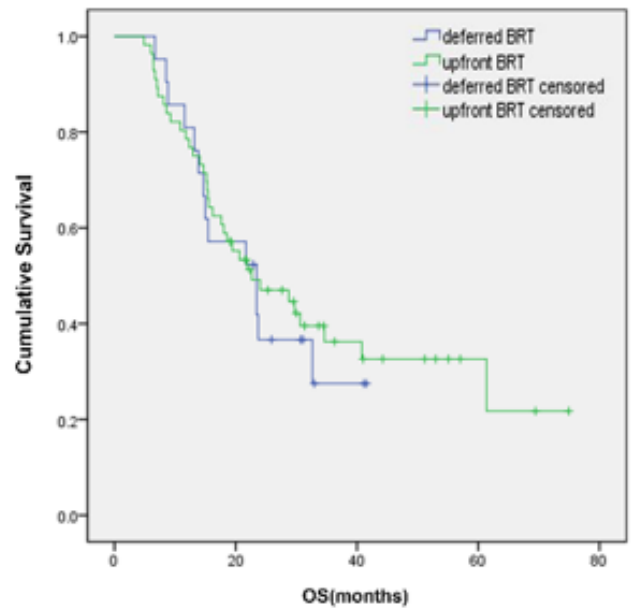

\section{Figure 2}

Kaplan-Meier survival curves for iPFS and OS in patient subgroups. (A) iPFS for patients with EGFR mutation; (B) OS for patients with EGFR mutation; (C) iPFS for patients without genetic testing results; (D) OS for patients without genetic testing results; (E) iPFS for patients without EGFR gene mutation; (F) OS for patients without EGFR gene mutation. Abbreviations: BRT, brain radiotherapy; iPFS, intracranial progression-free survival; OS, overall survival. 


\section{Supplementary Files}

This is a list of supplementary files associated with this preprint. Click to download.

- Appendix.docx 